\title{
Ceric Ammonium Nitrate Promoted Oxidation of Oxazoles
}

\author{
David A. Evans, Pavel Nagorny, and Risheng Xu \\ Department of Chemistry and Chemical Biology, Harvard University, Cambridge, MA, 02138
}

\section{Supporting Information}

\section{Contents}

1) General Information and Materials 2

2) Experimental Procedures and Analytical Data for Selected Oxazoles 3-10

3) Experimental Procedures and Analytical Data for Imides $11-21$

4) ${ }^{1} \mathrm{H}$ and ${ }^{13} \mathrm{C}$ Spectra of Selected Oxazoles $\quad 22-39$

5) ${ }^{1} \mathrm{H}$ and ${ }^{13} \mathrm{C}$ Spectra of Imides $\quad$ 40-79 


\section{General Information.}

All reactions were carried out under an atmosphere of dried nitrogen in flame-dried or oven-dried glassware with magnetic stirring, unless otherwise noted. Air-sensitive reagents and solutions were transferred via syringe or cannula and were introduced to the apparatus through rubber septa. Reactions were cooled via external cooling baths: ice water $\left(0^{\circ} \mathrm{C}\right)$, dry ice-acetone $\left(-78{ }^{\circ} \mathrm{C}\right)$, ice-acetone $(-10$ $\left.{ }^{\circ} \mathrm{C}\right)$, methanol-liquid nitrogen $\left(-95^{\circ} \mathrm{C}\right)$, or Neslab immersion cooler $\left(-20 \rightarrow-80{ }^{\circ} \mathrm{C}\right)$. Heating was accomplished by heating mantle or silicon oil bath using a temperature controller. Analytical thin layer chromatography (TLC) was performed on EM Reagent $0.25 \mathrm{~mm}$ silica gel 60-F plates. Visualization was accomplished with UV light and exposure to aqueous ceric ammonium molybdate (CAM) solution or anisaldehyde followed by heating. Flash chromatography was performed using EM silica gel 60 (230-240 mesh). Solvents for extraction and chromatography were HPLC grade.

When necessary, solvents and reagents were dried prior to use. Reagents were purified prior to use following the guidelines of Perrin and Armarego. Tetrahydrofuran (THF), dichloromethane $\left(\mathrm{CH}_{2} \mathrm{Cl}_{2}\right)$, toluene and diethyl ether $\left(\mathrm{Et}_{2} \mathrm{O}\right)$ were filtered through a column of activated alumina under an argon atmosphere. Methanol $(\mathrm{MeOH})$ was distilled from magnesium methoxide. Benzene, acetonitrile, 2,6lutidine, pyridine, $\quad \mathrm{N}, \mathrm{N}$-diisopropylethylamine, $\mathrm{N}, \mathrm{N}$-dimethylethylamine, triethylamine, diisopropylamine and chlorotrimethylsilane were distilled from calcium hydride.

Optical rotations were measured on a Jasco DIP-0181 digital polarimeter with a sodium lamp and reported as follows: $[\alpha]_{\mathrm{D}}(\mathrm{c} \mathrm{g} / 100 \mathrm{ml}$, solvent). Infrared spectra were recorded on a Perkin Elmer model 1600 FT-IR spectrometer. Mass spectra were obtained on a JEOL AX-505 or SX-102 high resolution magnetic sector mass spectrometer by the Harvard University Mass Spectrometry Laboratory. Electrospray mass spectra were obtained using a LCT mass spectrometer (Micromass Instruments, Beverly, MA). Exact mass measurements were obtained by internal calibration with an appropriate lock mass compound. ${ }^{1} \mathrm{H}$ NMR spectra were recorded on Varian Inova-600 (600 MHz), Inova-500 (500 $\mathrm{MHz}$ ) or Mercury-400 (400 MHz) spectrometers. Chemical shifts ( $\delta$ ) are reported from tetramethylsilane with the solvent resonance as the internal standard $\left(\mathrm{CDCl}_{3}: \delta 7.26, \mathrm{C}_{6} \mathrm{D}_{6}: \delta 7.15\right)$. Data are reported as follows: chemical shift $(\delta)$, multiplicity $(s=$ singlet, $d=$ doublet, $t=$ triplet, $q=$ quartet, $\mathrm{br}=$ broad, $\mathrm{m}=$ multiplet $)$, coupling constants $(\mathrm{Hz})$, integration, and assignment. ${ }^{13} \mathrm{C}$ NMR spectra were recorded on Varian INOVA-500 (125 MHz) or Mercury-400 (100 MHz) spectrometers with complete proton decoupling. Chemical shifts are reported in ppm from tetramethylsilane with the solvent as the internal standard $\left(\mathrm{CDCl}_{3}: \delta 77.0, \mathrm{C}_{6} \mathrm{D}_{6}: \delta 128.0\right)$. 


\section{General Procedure for the synthesis of 4,5 diphenyloxazoles.}

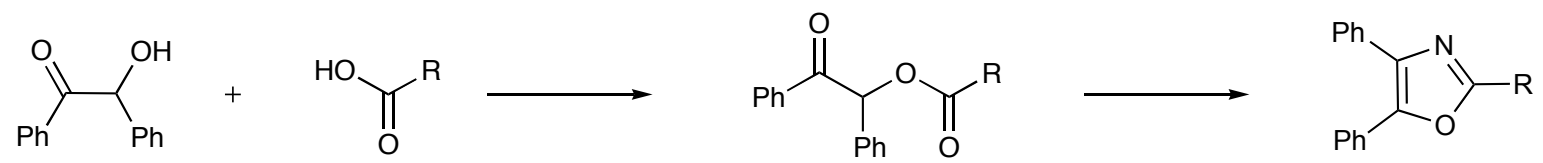

To a suitably sized, vacuum flame-dried RBF was added benzoin (1 equiv), dichloromethane $(0.2 \mathrm{M})$, dicyclohexylcarbodiimide (1 equiv), 4-dimethylaminopyridine (0.1 equiv), and a carboxylic acid of choice (1.1 equiv). The reaction was stirred for $6 \mathrm{~h}$ to overnight (until complete by TLC). The reaction was diluted with ethyl acetate and vacuum filtered through a silica gel plug. The resulting eluent was then washed with $5 \% \mathrm{HCl}$, saturated $\mathrm{NaHCO}_{3}$, brine, dried with $\mathrm{MgSO}_{4}$, filtered, and concentrated in vacuo. This crude mixture was then immediately purified by flash silica chromatography with the indicated solvents.

The mixture of an ester ( 1 equiv), ammonium acetate ( 5 equiv), and glacial acetic acid ( 0.685 M) was refluxed for 2 hours. The reaction was diluted with ethyl acetate and water. After extracting with ethyl acetate, the organic fractions were combined and washed with saturated $\mathrm{NaHCO}_{3}$, brine, dried with $\mathrm{MgSO}_{4}$, filtered, and concentrated in vacuo. This crude mixture was then immediately purified by flash chromatography with the indicated solvent mixtures.<smiles>CCc1nc(-c2ccccc2)c(-c2ccccc2)o1</smiles>

2-ethyl-4,5-diphenyloxazole (Table 1, Entry 1). 2-ethyl-4,5-diphenyloxazole was prepared by the previously reported procedure. ${ }^{1}$ The analytical data for the product matched the one reported in the cited reference.<smiles>CC(C)c1nc(-c2ccccc2)c(-c2ccccc2)o1</smiles>

2-isopropyl-4,5-diphenyloxazole (Table 1, Entry 2). 2-ethyl-4,5-diphenyloxazole was prepared by the previously reported procedure. ${ }^{1}$ The analytical data for the product matched the one reported in the cited reference.

(1) Pei, W.; Li, S.; Nie, X.; Li, Y.; Pei, J.; Chen, B.; Wu, J.; Ye, X. Synthesis 1998, 1298-1304. 


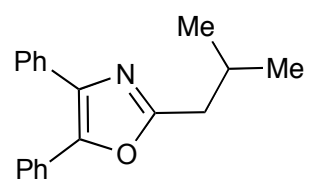

2-isobutyl-4,5-diphenyloxazole (Table 1, Entry 3). Benzoin (750 mg, 3.53 mmol), 4dimethylaminopyridine (43 mg, $0.35 \mathrm{mmol}$ ), and 3-methylbutanoic acid (397 mg, $3.88 \mathrm{mmol}$ ) were dissolved in dichloromethane $(17 \mathrm{~mL})$, and dicyclohexylcarbodiimide $(729 \mathrm{mg}, 3.53 \mathrm{mmol})$ was subsequently added. The reaction was allowed to stir for 8 hours. The reaction was worked up as indicated above, and purified via flash chromatography (silca, 10\% $\rightarrow 20 \% \mathrm{v} / \mathrm{v}$ ethyl acetate/hexanes elution). After concentration of the appropriate fractions $\left(\mathrm{R}_{f}=0.7,20 \% \mathrm{v} / \mathrm{v}\right.$ ethyl acetate/hexanes $), 2$ oxo-1,2-diphenylethyl 3-methylbutanoate was obtained (911 mg, 81\% yield) as an oil.

2-oxo-1,2-diphenylethyl 3-methylbutanoate $(911 \mathrm{mg}, 3.07 \mathrm{mmol})$, ammonium acetate $(1.18 \mathrm{~g}$, $15.4 \mathrm{mmol})$ and acetic acid $(4.5 \mathrm{~mL})$ were added to a $25 \mathrm{~mL}$ flask. The reaction was refluxed at $120^{\circ} \mathrm{C}$ for 2 hours. After work up, the reaction was purified via flash chromatography (silica, 15\% $\rightarrow 20 \%$ ethyl acetate/hexanes) and the appropriate fractions concentrated ( $\mathrm{R}_{f} 0.77,15 \%$ ethyl acetate/hexanes) to afford $681 \mathrm{mg}$ product in 80\% yield: IR (film): 3061, 2958, 2870, 1949, 1888, 1702, 1682, 1597 , 1567, 1503, 1464, 1446, 1369, 1302, 1265, 1206, 1175, 1058, 1025, 982, 962, $914 \mathrm{~cm}^{-1}$; ${ }^{1} \mathrm{H}-\mathrm{NMR}$ $\left(500 \mathrm{MHz}, \mathrm{CDCl}_{3}\right) \delta$ 7.65-7.66 (m, 2H), 7.58-7.60 (m, 2H), 7.31-7.38 (m, 6H), $2.74(\mathrm{~d}, J=7.5 \mathrm{~Hz}, 2 \mathrm{H})$, $2.58(\mathrm{dq}, J=6.5 \mathrm{~Hz}, 7.5 \mathrm{~Hz}, 1 \mathrm{H}), 1.06(\mathrm{~d}, J=6.5 \mathrm{~Hz}, 6 \mathrm{H}) ;{ }^{13} \mathrm{C}-\mathrm{NMR}\left(500 \mathrm{MHz}, \mathrm{CDCl}_{3}\right) \delta 163.2$, $145.1,135.0,132.7,129.2,128.9,128.6,128.5,128.3,128.0,126.4,37.1,27.7,22.5$; HRMS (ES+): Exact mass calcd for $\mathrm{C}_{17} \mathrm{H}_{15} \mathrm{NO}[\mathrm{M}+\mathrm{H}]^{+}: 278.1545$. Found: 278.1543 .

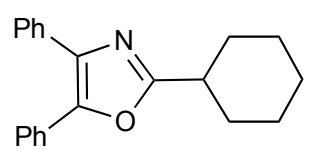

2-cyclohexyl-4,5-diphenyloxazole (Table 1, Entry 4). Benzoin (1.0 g, 4.7 mmol), 4dimethylaminopyridine (58 $\mathrm{mg}, 0.47 \mathrm{mmol})$, and cyclohexanecarboxylic acid (664 $\mathrm{mg}, 5.18 \mathrm{mmol})$ were dissolved in dichloromethane $(23.6 \mathrm{~mL})$, and dicyclohexylcarbodiimide $(972 \mathrm{mg}, 4.7 \mathrm{mmol})$ was subsequently added. The reaction was allowed to stir overnight. The reaction was worked up as indicated above, and purified via flash chromatography (silca, 10\% $\rightarrow 20 \% \mathrm{v} / \mathrm{v}$ ethyl acetate/hexanes elution). After concentration of the appropriate fractions $\left(\mathrm{R}_{f}=0.68,25 \% \mathrm{v} / \mathrm{v}\right.$ ethyl acetate/hexanes), this afforded $1.27 \mathrm{~g}$ of 2-oxo-1,2-diphenylethyl cyclohexanecarboxylate in (84\% yield).

2-oxo-1,2-diphenylethyl cyclohexanecarboxylate $(851 \mathrm{mg}, 2.64 \mathrm{mmol})$, ammonium acetate $(1.02 \mathrm{~g}, 13.2 \mathrm{mmol})$ and acetic acid $(1.02 \mathrm{~g})$ were added to a $25 \mathrm{~mL}$ flask. The reaction was refluxed at 
$120^{\circ} \mathrm{C}$ for 2 hours. After work up, the reaction was purified via flash chromatography (silica, $15 \% \rightarrow$ $20 \%$ ethyl acetate/hexanes) and concentration of the appropriate fractions $\left(\mathrm{R}_{f} 0.8,20 \%\right.$ ethyl acetate/hexanes) to afford $801 \mathrm{mg}$ product in quantitative yield: IR (film): 3056, 2930, 2853, 1955, 1880, 1604, 1566, 1501, 1446, 1219, 1059, 1025, $966 \mathrm{~cm}^{-1} ;{ }^{1} \mathrm{H}-\mathrm{NMR}\left(600 \mathrm{MHz}, \mathrm{CDCl}_{3}\right) \delta$ 7.64-7.66 (m, 2H), 7.57-7.59 (m, 2H), 7.31-7.38 (m, 6H), 2.88-2.93 (m, 1H), 2.14-2.17 (m, 2H), 1.85-1.88 (m, $2 \mathrm{H}), 1.68-1.75(\mathrm{~m}, 3 \mathrm{H}), 1.30-1.45(\mathrm{~m}, 3 \mathrm{H}) ;{ }^{13} \mathrm{C}-\mathrm{NMR}\left(100 \mathrm{MHz}, \mathrm{CDCl}_{3}\right) \delta 166.7,144.5,134.8,132.7$, 129.2, 128.5, 128.4, 128.1, 127.9, 127.8, 126.2, 37.5, 30.6, 25.7, 25.6; HRMS (ES+): Exact mass calcd for $\mathrm{C}_{21} \mathrm{H}_{21} \mathrm{NO}[\mathrm{M}+\mathrm{H}]^{+}:$304.1701. Found: 304.1699 .

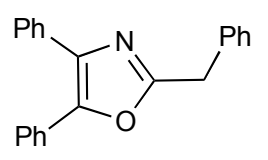

2-benzyl-4,5-diphenyloxazole (Table 1, Entry 5). Benzoin (l.0 $\mathrm{g}, 4.7$ mmol), $4-$ dimethylaminopyridine $(58 \mathrm{mg}, 0.47 \mathrm{mmol})$, and phenylacetic acid $(706 \mathrm{mg}, 5.18 \mathrm{mmol}$ ) were dissolved in dichloromethane $(23.56 \mathrm{~mL})$, and dicyclohexylcarbodiimide $(972 \mathrm{mg}, 4.70 \mathrm{mmol})$ was subsequently added. The reaction was allowed to stir for 6 hours. The reaction was worked up as indicated above, and purified via flash chromatography (silca, $10 \% \rightarrow 20 \% \mathrm{v} / \mathrm{v}$ ethyl acetate/hexanes elution). After concentration of the appropriate fractions $\left(\mathrm{R}_{f}=0.6,25 \% \mathrm{v} / \mathrm{v}\right.$ ethyl acetate/hexanes), this afforded $1.52 \mathrm{~g}$ of 2-oxo-1,2-diphenylethyl 2-phenylacetate in (98\% yield).

2-oxo-1,2-diphenylethyl 2-phenylacetate $(1.72 \mathrm{~g}, 5.22 \mathrm{mmol})$, ammonium acetate $(2.01 \mathrm{~g}, 26.1$ $\mathrm{mmol})$, and acetic acid $(7.6 \mathrm{~mL})$ were added to a $25 \mathrm{~mL}$ flask. The reaction was refluxed at $120{ }^{\circ} \mathrm{C}$ for 2 hours. After work up, the reaction was purified via flash chromatography (silica, $20 \% \rightarrow 30 \% \mathrm{v} / \mathrm{v}$ ethyl acetate/hexanes elution). After concentration of the appropriate fractions $\left(\mathrm{R}_{f} 0.8,15 \% \mathrm{v} / \mathrm{v}\right.$ ethyl acetate/hexanes) to afford $1.53 \mathrm{~g}$ title compound in 94\% yield: IR (film): 3061, 3030, 1681, 1603, 1567, 1496, 1447, 1218, 1058, 1025, $962 \mathrm{~cm}^{-1} ;{ }^{1} \mathrm{H}-\mathrm{NMR}\left(600 \mathrm{MHz}, \mathrm{CDCl}_{3}\right) \delta$ 7.29-7.69 (m, 15H), 4.24 (s, 2H); ${ }^{13} \mathrm{C}-\mathrm{NMR}\left(500 \mathrm{MHz}, \mathrm{CDCl}_{3}\right) \delta 161.5,145.6,135.3,135.0,132.2,129.7,128.7,128.6,128.4$, $128.3,127.9,127.8,126.9,126.3,34.5$; HRMS (ESI): Exact mass calcd for $\mathrm{C}_{22} \mathrm{H}_{17} \mathrm{NO}[\mathrm{M}+\mathrm{H}]^{+}$: 312.1388. Found: 312.1378 .

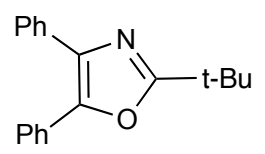

2-tert-butyl-4,5-diphenyloxazole (Table 1, Entry 6). Benzoin (1.0 g, $4.7 \mathrm{mmol})$ and 4dimethylaminopyridine (228 mg, $2.03 \mathrm{mmol})$ were dissolved in dichloromethane $(16 \mathrm{~mL})$ and pyridine $(7.6 \mathrm{~mL})$. Pivaloyl chloride $(625 \mathrm{mg}, 5.18 \mathrm{mmol})$ was subsequently added, and the reaction was 
allowed to stir for 2 hours. The reaction was worked up as indicated above, and purified via flash chromatography (silca, $10 \% \rightarrow 20 \% \mathrm{v} / \mathrm{v}$ ethyl acetate/hexanes elution). After concentration of the appropriate fractions $\left(\mathrm{R}_{f}=0.8,25 \% \mathrm{v} / \mathrm{v}\right.$ ethyl acetate/hexanes), this afforded $1.39 \mathrm{~g}$ of 2-oxo-1,2diphenylethyl pivalate in quantitative yield.

2-oxo-1,2-diphenylethyl pivalate (1.62 g, $5.47 \mathrm{mmol})$, ammonium acetate $(2.11 \mathrm{~g}, 27.4 \mathrm{mmol})$ and acetic acid $(8.0 \mathrm{~mL})$ were added to a $25 \mathrm{~mL}$ flask. The reaction was refluxed at $120^{\circ} \mathrm{C}$ for 2 hours. After work up, the reaction was purified via flash chromatography (silica, $10 \% \rightarrow 15 \%$ ethyl acetate/hexanes) and the appropriate fractions concentrated $\left(\mathrm{R}_{f} 0.7,50 \%\right.$ ethyl acetate/hexanes $)$ to afford 1.49 g product in 98\% yield: IR (film): 3059, 2971, 2870, 1948, 1886, 1808, 1754, 1605, 1565, 1502, 1444, 1369, 1301, 1227, 1178, 1127, 1059, 1025, $973 \mathrm{~cm}^{-1} ;{ }^{1} \mathrm{H}-\mathrm{NMR}\left(500 \mathrm{MHz}, \mathrm{CDCl}_{3}\right) \delta 7.65-$ $7.67(\mathrm{~m}, 2 \mathrm{H}), 7.59-7.61(\mathrm{~m}, 2 \mathrm{H}), 7.29-7.39(\mathrm{~m}, 6 \mathrm{H}), 1.49(\mathrm{~s}, 9 \mathrm{H}) ;{ }^{13} \mathrm{C}-\mathrm{NMR}\left(100 \mathrm{MHz}, \mathrm{CDCl}_{3}\right) \delta 169.8$, $144.7,134.9,132.9,129.4,128.6,128.5,128.2$, 128.1, 127.9, 126.3, 33.8, 28.7; HRMS (ES+): Exact mass calcd for $\mathrm{C}_{19} \mathrm{H}_{19} \mathrm{NO}[\mathrm{M}+\mathrm{H}]^{+}: 278.1545$. Found: 278.1533 .<smiles>c1ccc(-c2cnc(-c3ccccc3)o2)cc1</smiles>

4,5-diphenyloxazole (Table 1, Entry 7). 4,5-diphenyloxazole was prepared by the previously reported procedure. ${ }^{1}$ The analytical data for the product matched the one reported in the cited reference

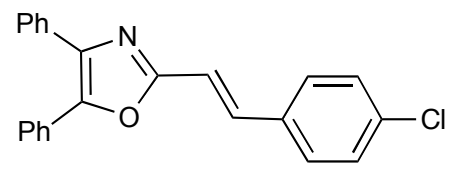

(E)-2-(4-chlorostyryl)-4,5-diphenyloxazole (Table 1, Entry 8). Benzoin (1.0 g, 4.7 mmol), 4-

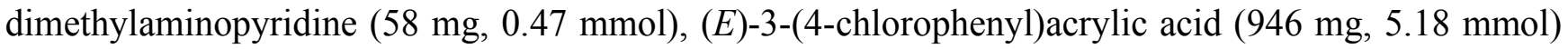
were dissolved in dichloromethane $(23.6 \mathrm{~mL})$, and dicyclohexylcarbodiimide $(972 \mathrm{mg}, 4.7 \mathrm{mmol})$ was subsequently added. The reaction was allowed to stir for 8 hours. The reaction was worked up as indicated above, and purified via flash chromatography ( silca, 10\% $\rightarrow 20 \% \mathrm{v} / \mathrm{v}$ ethyl acetate/hexanes elution). After concentration of the appropriate fractions $\left(\mathrm{R}_{f}=0.7,20 \% \mathrm{v} / \mathrm{v}\right.$ ethyl acetate/hexanes $)$, this afforded $1.13 \mathrm{~g}$ of (E)-2-oxo-1,2-diphenylethyl 3-(4-chlorophenyl)acrylate in 64\% yield.

(E)-2-oxo-1,2-diphenylethyl 3-(4-chlorophenyl)acrylate (825.4 $\mathrm{mg}, 2.19 \mathrm{mmol})$, ammonium acetate $(844.3 \mathrm{mg}, 10.95 \mathrm{mmol})$ and acetic acid $(3.2 \mathrm{~mL})$ were added to a $25 \mathrm{~mL}$ flask. The reaction was refluxed at $120^{\circ} \mathrm{C}$ for 2 hours. After work up, the reaction was purified via flash chromatography (silica, $10 \% \rightarrow 40 \%$ ethyl acetate/hexanes) and the appropriate fractions concentrated $\left(\mathrm{R}_{f} 0.6,20 \%\right.$ 
ethyl acetate/hexanes) to afford $578 \mathrm{mg}$ product in 74\% yield: IR (film): 3059, 2930, 2855, 1953, 1894, 1825, 1699, 1636, 1591, 1531, 1489, 1444, 1405, 1323, 1177, 1090, 1068, 1012, $963 \mathrm{~cm}^{-1}$; ${ }^{1} \mathrm{H}-\mathrm{NMR}$ $\left(600 \mathrm{MHz}, \mathrm{CDCl}_{3}\right) \delta$ 7.72-7.74 (m, 2H), 7.69-7.71 (m, 2H), $7.59(\mathrm{~d}, J=16.2 \mathrm{~Hz}, 1 \mathrm{H}), 7.52-7.53(\mathrm{~m}$, 2H), 7.39-7.46 (m, 8H), $7.03(\mathrm{~d}, J=16.2 \mathrm{~Hz}, 1 \mathrm{H}) ;{ }^{13} \mathrm{C}-\mathrm{NMR}\left(100 \mathrm{MHz}, \mathrm{CDCl}_{3}\right) \delta 159.5,145.2,136.8$, $134.6,134.4,133.9,132.2,129.2$, 128.9, 128.8, 128.5, 128.4, 128.1, 127.8, 126.3, 114.0; HRMS (ES+): Exact mass calcd for $\mathrm{C}_{23} \mathrm{H}_{16} \mathrm{ClNO}[\mathrm{M}+\mathrm{H}]+:$ 358.0998. Found: 358.0986.

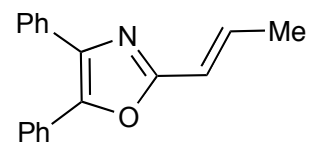

(E)-4,5-diphenyl-2-(prop-1-enyl)oxazole (Table 1, Entry 9). Benzoin (1.0 g, 4.7 mmol), 4dimethylaminopyridine (58 $\mathrm{mg}, 0.47 \mathrm{mmol}$ ), and $(E)$-but-2-enoic acid (446 $\mathrm{mg}, 5.2 \mathrm{mmol}$ ) were dissolved in dichloromethane $(23.6 \mathrm{~mL})$, and dicyclohexylcarbodiimide $(970 \mathrm{mg}, 4.70 \mathrm{mmol})$ was subsequently added. The reaction was allowed to stir overnight. The reaction was worked up as indicated above, and purified via flash chromatography (silca, 10\% $\rightarrow 20 \% \mathrm{v} / \mathrm{v}$ ethyl acetate/hexanes elution). After concentration of the appropriate fractions $\left(\mathrm{R}_{f}=0.7,25 \% \mathrm{v} / \mathrm{v}\right.$ ethyl acetate/hexanes $)$, this afforded $988 \mathrm{mg}$ of $(E)$-2-oxo-1,2-diphenylethyl but-2-enoate in (75\% yield).

(E)-2-oxo-1,2-diphenylethyl but-2-enoate $(830 \mathrm{mg}, 2.96 \mathrm{mmol})$, ammonium acetate (1.14 g, 14.8 mmol) and acetic acid $(4.32 \mathrm{~mL})$ were added to a $25 \mathrm{~mL}$ flask. The reaction was refluxed at $120{ }^{\circ} \mathrm{C}$ for 2 hours. After work up, the reaction was purified via flash chromatography (silica, 10\% $\rightarrow 15 \%$ ethyl acetate/hexanes) and the appropriate fractions concentrated $\left(\mathrm{R}_{f} 0.8,20 \%\right.$ ethyl acetate/hexanes $)$ to afford $742 \mathrm{mg}$ product in 94\% yield: IR (film): 3055, 2969, 2928, 2850, 2358, 1665, 1602, 1536, 1495, 1481, 1444, 1372, 1318, 1211, 1063, 1025, $962 \mathrm{~cm}^{-1} ;{ }^{1} \mathrm{H}-\mathrm{NMR}\left(500 \mathrm{MHz}, \mathrm{CDCl}_{3}\right) \delta 7.75-7.76(\mathrm{~m}, 2 \mathrm{H})$, 7.67-7.68 (m, 2H), 7.32-7.43 (m, 6H), 6.86-6.92 (m, 1H), $6.45(\mathrm{dd}, J=16.2 \mathrm{~Hz}, 1.8 \mathrm{~Hz}), 1.99(\mathrm{dd}, J=$ $6.6 \mathrm{~Hz}, 1.8 \mathrm{~Hz}, 3 \mathrm{H}) ;{ }^{13} \mathrm{C}-\mathrm{NMR}\left(500 \mathrm{MHz}, \mathrm{CDCl}_{3}\right) \delta 159.5,144.4,135.8,135.3,132.3,126.6,128.8$, 128.7, 128.4, 128.3, 128.2, 127.9, 127.8, 126.2, 117.5, 18.3; HRMS (ES+): Exact mass calcd for $\mathrm{C}_{18} \mathrm{H}_{15} \mathrm{NO}[\mathrm{M}+\mathrm{H}]^{+}: 262.1232$. Found: 262.1230 .

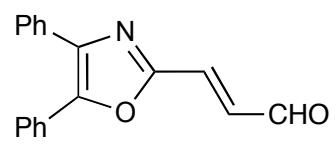

(E)-3-(4,5-diphenyloxazol-2-yl)acrylaldehyde (Table 1, Entry 10). (E)-3-(4,5-diphenyloxazol-2yl)acrylaldehyde was prepared by the previously reported procedure. ${ }^{2}$

(2) Evans, D. A.; Nagorny, P.; Reynolds, D. J.; McRae, K. J. Angew. Chem. Int. Ed. 2006, 45, In Press. 


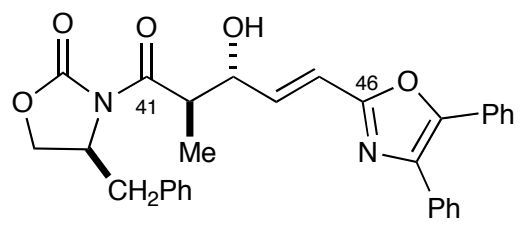

(S)-4-benzyl-3-((2R,3R,E)-5-(4,5-diphenyloxazol-2-yl)-3-hydroxy-2-methylpent-4-

enoyl)oxazolidin-2-one (Table 1, Entry 11). (S)-4-benzyl-3-((2R,3R,E)-5-(4,5-diphenyloxazol-2-yl)3-hydroxy-2-methylpent-4-enoyl)oxazolidin-2-one was prepared by the previously reported procedure. ${ }^{2}$<smiles>CC(CCc1nc(-c2ccccc2)c(-c2ccccc2)o1)[C@@H](O)C(=O)N1C(=O)OC[C@H]1C</smiles>

(S)-4-benzyl-3-((2R,3R)-5-(4,5-diphenyloxazol-2-yl)-3-hydroxy-2-methylpentanoyl)oxazolidin-2one (Table 1, Entry 12). (S)-4-benzyl-3-((2R,3R)-5-(4,5-diphenyloxazol-2-yl)-3-hydroxy-2methylpentanoyl)oxazolidin-2-one was prepared by the previously reported procedure. ${ }^{2}$<smiles>O=Cc1oc(-c2ccccc2)nc1-c1ccccc1</smiles>

2,4-diphenyloxazole-5-carbaldehyde (Table 3, Entry 2). 2,4-diphenyloxazole (200 mg, $0.904 \mathrm{mmol}$ ) was dissolved in THF $(9 \mathrm{~mL}, 0.1 \mathrm{M})$ and cooled to $-78^{\circ} \mathrm{C} . n-\mathrm{BuLi}(0.402 \mathrm{~mL}$, titrated at $2.7 \mathrm{M})$ was added dropwise over 5 minutes, and the reaction was stirred for additional 15 minutes. $N, N$ dimethylformamide $(1.5 \mathrm{~mL})$ was subsequently added dropwise, and the obtained solution was allowed to warm up to room temperature over $3 \mathrm{~h}$ period. The reaction was then quenched with $\mathrm{NH}_{4} \mathrm{Cl}_{\text {(sat.), }}$, extracted with EtOAc $(3 \times 5 \mathrm{~mL})$, and washed with saturated solutions of $\mathrm{NaHCO}_{3}$ and $\mathrm{NaCl}$. The organic fraction was then dried with $\mathrm{MgSO}_{4}$, filtrated, and concentrated in vacuo. The crude mixture was subjected to flash chromatography (silica 1\% ethyl acetate/hexanes) and the concentration of the appropriate fractions ( $\mathrm{R}_{f}=0.8,5 \%$ ethyl acetate/hexanes), afforded the title compound $(180 \mathrm{mg}, 80 \%)$ : IR (film): 3448, 3065, 2851, 1668, 1606, 1545, 1448, 1372, 1288, 1233, 1158, 1072, 1000, $806 \mathrm{~cm}^{-1}$; ${ }^{1} \mathrm{H}-\mathrm{NMR}\left(600 \mathrm{MHz}, \mathrm{CDCl}_{3}\right) \delta 10.00(\mathrm{~s}, 1 \mathrm{H}), 8.25-8.26(\mathrm{~m}, 2 \mathrm{H}), 8.04-8.05(\mathrm{~m}, 2 \mathrm{H}), 7.52-7.59(\mathrm{~m}, 6 \mathrm{H})$; ${ }^{13} \mathrm{C}-\mathrm{NMR}\left(100 \mathrm{MHz}, \mathrm{CDCl}_{3}\right) \delta 177.0,164.0,151.5,144.3,132.2,130.6,129.6,128.9,128.8,127.8$, 125.8; HRMS (ES+): Exact mass calcd for $\mathrm{C}_{16} \mathrm{H}_{11} \mathrm{NO}_{2}[\mathrm{M}+\mathrm{H}]^{+}: 250.0868$. Found: 250.0874 . 
<smiles>Cc1nc(C)c(C)o1</smiles>

2,5-dimethyl-4-phenyloxazole (Table 3, Entry 3). 2,5-dimethyl-4-phenyloxazole was prepared by the previously reported procedure. ${ }^{3}$<smiles>CCOC(=O)c1coc(/C=C/c2ccccc2)n1</smiles>

(E)-ethyl 2-styryloxazole-4-carboxylate (Table 3, Entry 4). 2-styryloxazole-4-carboxylate was prepared by the previously reported procedure. ${ }^{4 a}$ The analytical data for the product matched the one reported in the cited references.<smiles>CCC(=O)O[C@@H](CC(=O)N1C(=O)OCC1Cc1ccccc1)c1coc(C)n1</smiles>

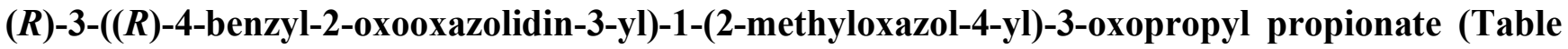
3, Entry 5). (R)-4-benzyl-3-((R)-3-hydroxy-3-(2-methyloxazol-4-yl)propanoyl)oxazolidin-2-one ${ }^{5}$ (200 $\mathrm{mg}, 0.605 \mathrm{mmol})$ was dissolved in DCM $(6 \mathrm{~mL})$ and triethylamine $(0.105 \mathrm{~mL})$. The reaction was cooled to $0{ }^{\circ} \mathrm{C}$ and propionyl chloride $(0.060 \mathrm{~mL})$ was added dropwise. The reaction was allowed to warm up to room temperature over 2 hours. The reaction was then quenched with water, extracted three times with EtOAc, and washed with saturated solutions of $\mathrm{NaHCO}_{3}$ and $\mathrm{NaCl}$. The organic fraction was then dried with $\mathrm{MgSO}_{4}$, vacuum filtered, and concentrated in vacuo. The crude mixture was subjected to flash chromatography (silica, $50 \% \mathrm{v} / \mathrm{v}$ ethyl acetate/hexanes), and the appropriate fractions concentrated $\left(\mathrm{R}_{f}=0.5,50 \% \mathrm{v} / \mathrm{v}\right.$ ethyl acetate/hexanes) to afford $173 \mathrm{mg}$ of the title compound in $74 \%$ yield: $[\alpha]_{\mathrm{D}}^{25}=-14.6\left(c=2.46, \mathrm{CHCl}_{3}\right)$; IR (film) 2982, 2943, 1781, 1738, 1700, 1583, 1490, 1454, 1391, 1353, 1210, 1180, 1106, 1080, 1998, $919 \mathrm{~cm}^{-1} ;{ }^{1} \mathrm{H}-\mathrm{NMR}\left(500 \mathrm{MHz}, \mathrm{CDCl}_{3}\right) \delta 7.55$ (s, 1H), 7.30$7.33(\mathrm{~m}, 2 \mathrm{H}), 7.26-7.27(\mathrm{~m}, 1 \mathrm{H}), 7.18-7.19(\mathrm{~m}, 2 \mathrm{H}), 6.30(\mathrm{t}, J=6.5 \mathrm{~Hz}, 1 \mathrm{H}), 4.61-4.64(\mathrm{~m}, 1 \mathrm{H}), 4.21$

(3) (a) Nagayoshi, K.; Sato, T. Chem. Lett. 1983, 9, 1355-1356. (b) Milton, M. D.; Inada, Y.; Nishibayashi, Y.; Uemura, S. Chem. Comm. 2004, 23, 2712-2713.

(4) (a) Panek, J. S.; Beresis, R. T. J. Org. Chem. 1996, 61, 6496-6497. (b) Evans, D. A.; Fitch, D. M.; Smith, T. E.; Cee, V. J.; Cho, P. S. J. Am. Chem. Soc. 2000, 122, 10033-10046. (c) Boger, D. L.; Curran, T. T. J. Org. Chem. 1992, 57, 2235-2244.

(5) This compound was synthesized similar to

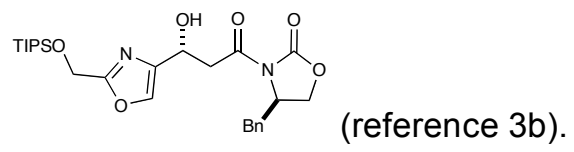


(t, $J=8,1 \mathrm{H}), 4.15(\mathrm{dd}, J=3 \mathrm{~Hz}, 6 \mathrm{~Hz}), 4.61(\mathrm{dd}, J=2 \mathrm{~Hz}, 4.5 \mathrm{~Hz}), 3.25(\mathrm{dd}, J=9 \mathrm{~Hz}, 13 \mathrm{~Hz}), 2.42(\mathrm{~s}$, $1 \mathrm{H}), 2.33(\mathrm{~m}, 2 \mathrm{H}), 1.12(\mathrm{t}, J=8 \mathrm{~Hz}, 3 \mathrm{H}) ;{ }^{13} \mathrm{C}-\mathrm{NMR}\left(100 \mathrm{MHz}, \mathrm{CDCl}_{3}\right) \delta 173.5,169.3,161.8,153.3$, 138.3, 136.1, 135.0, 129.4, 128.9, 127.3, 66.2, 63.9, 55.0, 39.5, 37.6, 27.5, 14.9, 8.9; HRMS (ES+): Exact mass calcd for $\mathrm{C}_{20} \mathrm{H}_{22} \mathrm{~N}_{2} \mathrm{O}_{6}[\mathrm{M}+\mathrm{H}]^{+}: 387.1556$. Found: 387.1570 .

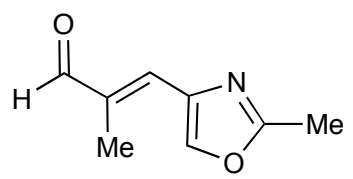

(E)-2-methyl-3-(2-methyloxazol-4-yl)acrylaldehyde (Table 3, Entry 6). (E)-2-methyl-3-(2methyloxazol-4-yl)acrylaldehyde was prepared by the previously reported procedure. ${ }^{4 \mathrm{~b}, 4 \mathrm{c}}$ The analytical data for the product matched the one reported in the cited reference. 


\section{General Procedure for the CAN oxidation of oxazoles.}

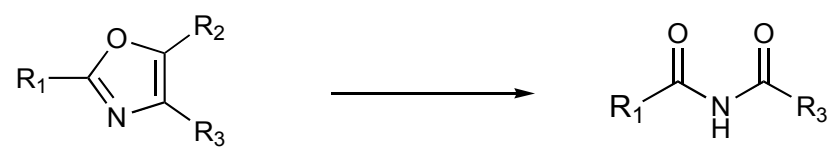

To the solution of an appropriate oxazole (1 equiv) in $\mathrm{MeCN} / \mathrm{H}_{2} \mathrm{O}(9: 1,0.06 \mathrm{M})$, ceric ammonium nitrate (2-4 equiv) was added. The reaction was allowed to stir until completion via TLC and then diluted with ethyl acetate and water. After extracting with ethyl acetate, the organic fractions were combined, washed with $\mathrm{NaHCO}_{3}$ (sat.) and brine, dried with $\mathrm{MgSO}_{4}$, filtered, and concentrated in vacuo. This crude mixture was then immediately purified by flash chromatography.

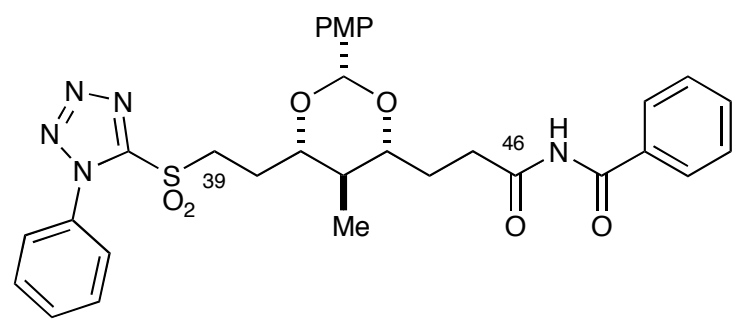

\section{$N$-(3-((2R,4R,5R,6S)-2-(4-methoxyphenyl)-5-methyl-6-(2-(1-phenyl-1H-tetrazol-5-}

ylsulfonyl)ethyl)-1,3-dioxan-4-yl)propanoyl)benzamide (Scheme 1, 2). To the solution of oxazole $\mathbf{1}^{2}(25.0 \mathrm{mg}, 37.4 \mathrm{mmol})$ in acetonitrile/water mixture $(9: 1,1.0 \mathrm{~mL})$ CAN (63.3 $\left.\mathrm{mg}, 116 \mathrm{mmol}\right)$ was added. The obtained orange solution was stirred for $1.5 \mathrm{~h}$ before being treated with water $(5 \mathrm{~mL})$ and ethyl acetate $(5 \mathrm{~mL})$. The aqueous phase was extracted with ethyl acetate $(2 \times 2 \mathrm{~mL})$, and the combined organic phase was washed with $\mathrm{K}_{2} \mathrm{CO}_{3 \text { (sat.) }}(5 \mathrm{~mL})$, brine $(3 \mathrm{~mL})$, dried $\left(\mathrm{MgSO}_{4}\right)$, filtered and concentrated under reduced pressure to afford a white solid. The purification of the crude product by flash chromatography (silica, 50\% v/v ethyl acetate/hexanes elution) afforded, after concentration of the appropriate fractions $\left(\mathrm{R}_{f} 0.2,33 \% \mathrm{v} / \mathrm{v}\right.$ ethyl acetate/hexanes), the title compound $2(16.0 \mathrm{mg}, 76 \%)$ as a white foam: $[\alpha]_{\mathrm{D}}{ }^{25}=+12\left(c=0.61, \mathrm{CHCl}_{3}\right)$; IR (film) 3260, 3072, 2966, 2931, 2837, 1725, 1707, $1684,1672,1613,1496,1460,1402,1343,1178,1149,1126,1073,1031 \mathrm{~cm}^{-1} ;{ }^{1} \mathrm{H}$ NMR $(500 \mathrm{MHz}$, $\left.\mathrm{CDCl}_{3}\right) \delta 8.48(\mathrm{~s}, \mathrm{NH}), 7.75(\mathrm{~d}, J=8.8 \mathrm{~Hz}, 2 \mathrm{H}), 7.65(\mathrm{~d}, J=8.6 \mathrm{~Hz}, 2 \mathrm{H}), 7.62-7.56(\mathrm{~m}, 4 \mathrm{H}), 7.47(\mathrm{t}, J$ $=8.3 \mathrm{~Hz}, 2 \mathrm{H}), 7.37(\mathrm{~d}, J=8.3 \mathrm{~Hz}, 2 \mathrm{H}), 6.86(\mathrm{~d}, J=8.8 \mathrm{~Hz}, 2 \mathrm{H}), 5.47$ (s, 1H), 3.97 (ddd, $J=15.1,10.2$, $5.4 \mathrm{~Hz}, 1 \mathrm{H}), 3.86(\mathrm{~m}, 1 \mathrm{H}), 3.79(\mathrm{~s}, 3 \mathrm{H}), 3.64(\mathrm{td}, J=9.3,2.9 \mathrm{~Hz}, 1 \mathrm{H}), 3.57$ (td, $J=9.3,2.4 \mathrm{~Hz}, 1 \mathrm{H})$, $3.24(\mathrm{ddd}, J=14.2,8.4,5.9 \mathrm{~Hz}, 1 \mathrm{H}), 3.14(\mathrm{dt}, J=17.6,6.8 \mathrm{~Hz}, 1 \mathrm{H}), 2.50(\mathrm{~m}, 1 \mathrm{H}), 2.26(\mathrm{dt}, J=12.4$, $6.4 \mathrm{~Hz}, 1 \mathrm{H}), 2.14(\mathrm{~m}, 1 \mathrm{H}), 2.14(\mathrm{~m}, 1 \mathrm{H}), 1.63(\mathrm{dq}, J=17.0,6.8 \mathrm{~Hz}, 1 \mathrm{H}), 0.92(\mathrm{~d}, J=6.4 \mathrm{~Hz}, 3 \mathrm{H}) ;{ }^{13} \mathrm{C}$ NMR $\left(125 \mathrm{MHz}, \mathrm{CDCl}_{3}\right) \delta 175.9,165.4,160.0,153.5,133.2,131.5,130.7,130.2,129.7,129.0,128.5$, 
127.6, 127.4, 125.1, 113.6, 100.4, 80.7, 79.3, 55.3, 52.6, 38.6, 33.4, 27.3, 25.9, 12.1; HRMS (ES+): Exact mass calcd for $\mathrm{C}_{31} \mathrm{H}_{33} \mathrm{~N}_{5} \mathrm{O}_{7} \mathrm{~S}[\mathrm{M}+\mathrm{H}]^{+}: 620.2179$. Found: 620.2195 .

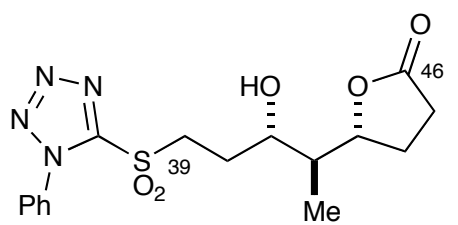

\section{(R)-5-((2S,3S)-3-hydroxy-5-(1-phenyl-1 $H$-tetrazol-5-ylsulfonyl)pentan-2-yl)-dihydrofuran-2(3H)-} one (Scheme 1, 3a). To the solution of oxazole $\mathbf{1}^{2}(28.3 \mathrm{mg}, 41.0 \mathrm{mmol})$ in acetonitrile/water mixture (8:1, $0.68 \mathrm{~mL}$ ) CAN (130 mg, $237 \mathrm{mmol})$ was added. The obtained solution was stirred for $35 \mathrm{~h}$ before being treated with water $(4 \mathrm{~mL})$ and ethyl acetate $(4 \mathrm{~mL})$. The aqueous phase was extracted with ethyl acetate $(2 \times 5 \mathrm{~mL})$, and the combined organic phase was washed with brine $(3 \mathrm{~mL})$, dried $\left(\mathrm{MgSO}_{4}\right)$, filtered and concentrated under reduced pressure to afford a white solid. The purification of the crude product by flash chromatography (silica, $80 \% \rightarrow 50 \% \mathrm{v} / \mathrm{v}$ methelene chloride/diethyl ether elution) afforded, after concentration of the appropriate fractions $\left(\mathrm{R}_{f} 0.2,67 \% \mathrm{v} / \mathrm{v}\right.$ methelene chloride/diethyl ether), the title compound 3a $(12.0 \mathrm{mg}, 77 \%)$ as a colorless oil: $[\alpha]_{\mathrm{D}}{ }^{25}=-30\left(c=0.65, \mathrm{CHCl}_{3}\right)$; IR (film) $v_{\max } 3437,2967,2931,2850,1765,1593,1340,1269,1224,1188,1151 \mathrm{~cm}^{-1} ;{ }^{1} \mathrm{H}$ NMR $(500$ $\left.\mathrm{MHz}, \mathrm{CDCl}_{3}\right) \delta 7.68(\mathrm{~d}, J=6.9 \mathrm{~Hz}, 2 \mathrm{H}), 7.62-7.59(\mathrm{~m}, 3 \mathrm{H}), 4.45$ (td, $\left.J=9.2,6.4 \mathrm{~Hz}, 1 \mathrm{H}\right), 3.99$ (ddd, $J$ $=14.6,9.6,4.6 \mathrm{~Hz}, 1 \mathrm{H}), 3.87-3.76(\mathrm{~m}, 2 \mathrm{H}), 2.52(\mathrm{~d}, J=10.5 \mathrm{~Hz}, 1 \mathrm{H}), 2.51(\mathrm{~d}, J=10.1 \mathrm{~Hz}, 1 \mathrm{H}), 2.32$ (dq, $J=12.4,6.4 \mathrm{~Hz}, 2 \mathrm{H}), 2.44$ (tdd, $J=9.2,6.0,2.7 \mathrm{~Hz}, 1 \mathrm{H}), 2.08$ (dtd, $J=14.6,9.6,5.0 \mathrm{~Hz}, 1 \mathrm{H}$ ), 1.96-1.89 (m, 2H), $0.92(\mathrm{~d}, J=6.9 \mathrm{~Hz}, 3 \mathrm{H}) ;{ }^{13} \mathrm{C} \mathrm{NMR}\left(125 \mathrm{MHz}, \mathrm{CDCl}_{3}\right) \delta$ 176.3, 153.6, 133.0, 131.5, 129.8, 125.2, 82.5, 71.5, 53.2, 44.0, 28.4, 26.8, 26.6, 10.7; HRMS (ES+): Exact mass calcd for $\mathrm{C}_{16} \mathrm{H}_{20} \mathrm{~N}_{4} \mathrm{O}_{5} \mathrm{~S}[\mathrm{M}+\mathrm{H}]^{+}: 381.1232$. Found: 381.1234 .

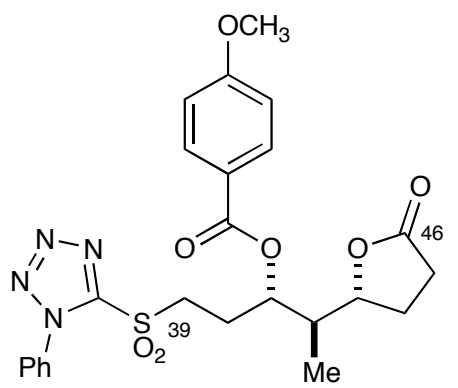

(R)-5-((2S,3S)-5-(4-methoxyphenyl)-5-oxo-3-(2-(1-phenyl-1H-tetrazol-5-ylsulfonyl)ethyl)pentan2-yl)-dihydrofuran-2(3H)-one (Scheme $\mathbf{1}, \mathbf{3 b})$. To the solution of oxazole $\mathbf{1}^{\mathbf{2}}$ (24.7 $\left.\mathrm{mg}, 35.7 \mathrm{mmol}\right)$ in 
Page-13

methylene chloride/water mixture (9:1,1.0 mL) DDQ (37.2 $\mathrm{mg}, 167 \mathrm{mmol})$ was added. The obtained solution was stirred for $30 \mathrm{~h}$ before being treated with $\mathrm{NaHCO}_{3 \text { (sat.) }}(1 \mathrm{~mL})$ and $\mathrm{Na}_{2} \mathrm{SO}_{3}(1 \mathrm{M}, 1 \mathrm{~mL})$. The aqueous phase was extracted with ethyl acetate $(2 \times 5 \mathrm{~mL})$, and the combined organic phase was washed with brine $(3 \mathrm{~mL})$, dried $\left(\mathrm{MgSO}_{4}\right)$, filtered and concentrated under reduced pressure to afford an orange oil. The purification of the crude product by flash chromatography (silica, $50 \% \mathrm{v} / \mathrm{v}$ ethyl acetate/hexanes elution) afforded, after concentration of the appropriate fractions $\left(\mathrm{R}_{f} 0.25,50 \% \mathrm{v} / \mathrm{v}\right.$ ethyl acetate/hexanes), the title compound $\mathbf{3 b}(13.0 \mathrm{mg}, 71 \%)$ as a white foam: $[\alpha]_{\mathrm{D}}{ }^{25}=-11(c=0.60$, $\mathrm{CHCl}_{3}$ ); IR (film) 3084, 2990, 2931, 2849, 1772, 1702, 1608, 1514, 1467, 1420, 1343, 1255, 1161, 1149, 1096, $1020 \mathrm{~cm}^{-1} ;{ }^{1} \mathrm{H}$ NMR $\left(500 \mathrm{MHz}, \mathrm{CDCl}_{3}\right) \delta 8.01(\mathrm{~d}, J=9.2 \mathrm{~Hz}, 2 \mathrm{H}), 7.67(\mathrm{~d}, J=6.4 \mathrm{~Hz}$, 2H), 7.61 (t, $J=6.4 \mathrm{~Hz}, 1 \mathrm{H}), 7.60(\mathrm{~d}, J=7.3 \mathrm{~Hz}, 2 \mathrm{H}), 6.96(\mathrm{~d}, J=8.9 \mathrm{~Hz}, 2 \mathrm{H}), 5.38$ (q, $J=5.5 \mathrm{~Hz}$, 1H), 4.47 (ddd, $J=9.2,7.8,6.4 \mathrm{~Hz}, 1 \mathrm{H}), 3.79$ (s, 3H), 3.87-3.76 (m, 2H), $2.54(\mathrm{~d}, J=10.1 \mathrm{~Hz}, 1 \mathrm{H})$, $3.53(\mathrm{~d}, J=10.1 \mathrm{~Hz}, 1 \mathrm{H}), 2.44$ (q, $J=6.4 \mathrm{~Hz}, 2 \mathrm{H}), 2.36$ (tq, $J=12.4,6.9 \mathrm{~Hz}, 1 \mathrm{H}), 2.29$ (dq, $J=12.8$, $6.4 \mathrm{~Hz}, 1 \mathrm{H}), 1.97(\mathrm{qd}, J=12.8,10.1 \mathrm{~Hz}, 1 \mathrm{H}), 1.02(\mathrm{~d}, J=6.9 \mathrm{~Hz}, 3 \mathrm{H}) ;{ }^{13} \mathrm{C} \mathrm{NMR}\left(125 \mathrm{MHz}, \mathrm{CDCl}_{3}\right)$ $\delta$ 176.3, 165.5, 164.0, 153.2, 133.9, 131.9, 131.5, 129.8, 125.1, 121.5, 114.0, 80.4, 72.4, 55.6, 53.2, 41.1, 28.6, 25.8, 24.5, 10.3; HRMS (ES+): Exact mass calcd for $\mathrm{C}_{24} \mathrm{H}_{26} \mathrm{~N}_{4} \mathrm{O}_{7} \mathrm{~S}[\mathrm{M}+\mathrm{H}]^{+}: 515.1600$. Found: 515.1611.<smiles>CCC(=O)NC(=O)c1ccccc1</smiles>

$\boldsymbol{N}$-propionylbenzamide (Table 1, Entry 1). 2-ethyl-4,5-diphenyloxazole (125 mg, $0.501 \mathrm{mmol}$ ) was dissolved in $\mathrm{MeCN}(7.5 \mathrm{~mL})$ and $\mathrm{H}_{2} \mathrm{O}(0.84 \mathrm{~mL})$, and $\mathrm{CAN}(1.04 \mathrm{~g}, 1.91 \mathrm{mmol})$ was added subsequently. The reaction was allowed to stir at room temperature for 2 hours. After work up, the reaction was purified via flash chromatography (silica $10 \% \rightarrow 25 \% \mathrm{v} / \mathrm{v}$ ethyl acetate/hexanes), and the appropriate fractions concentrated $\left(\mathrm{R}_{f}=0.4,25 \% \mathrm{v} / \mathrm{v}\right.$ ethyl acetate/hexanes) to afford $67 \mathrm{mg}$ of the title compound in 75\% yield: IR (film) 3287, 2975, 2937, 1677, 1600, 1584, 1506, 1468, 1367, 1239, 1206, 1076, 900, 811, $\mathrm{cm}^{-1} ;{ }^{1} \mathrm{H}-\mathrm{NMR}\left(600 \mathrm{MHz}, \mathrm{CDCl}_{3}\right) \delta 9.20(\mathrm{~s}, 1 \mathrm{H}), 7.90-7.92(\mathrm{~m}, 2 \mathrm{H}), 7.57-7.62(\mathrm{~m}$, $1 \mathrm{H}), 7.47-7.50(\mathrm{~m}, 2 \mathrm{H}), 3.02(\mathrm{q}, J=7.2,2 \mathrm{H}), 1.20(\mathrm{t}, J=7.2,3 \mathrm{H}) ;{ }^{13} \mathrm{C}-\mathrm{NMR}\left(100 \mathrm{MHz}, \mathrm{CDCl}_{3}\right) \delta$ 177.6, 165.7, 133.1, 132.8, 128.8, 127.9, 127.7, 31.2, 8.2; HRMS (ES+): Exact mass calcd for $\mathrm{C}_{10} \mathrm{H}_{11} \mathrm{NO}_{2}[\mathrm{M}+\mathrm{H}]^{+}:$178.0868. Found: 178.0860 .<smiles>CC(C)C(=O)NC(=O)c1ccccc1</smiles> 
$N$-isobutyrylbenzamide (Table 1, Entry 2). 2-isopropyl-4,5-diphenyloxazole (150 $\mathrm{mg}, 0.570 \mathrm{mmol})$ was dissolved in $\mathrm{MeCN}(8.5 \mathrm{~mL})$ and $\mathrm{H}_{2} \mathrm{O}(0.95 \mathrm{~mL})$, and CAN (1.19 g, $\left.2.16 \mathrm{mmol}\right)$ was added subsequently. The reaction was allowed to stir at room temperature for 2 hours. After work up, the reaction was purified via flash chromatography (silica, $10 \% \rightarrow 25 \% \mathrm{v} / \mathrm{v}$ ethyl acetate/hexanes) and appropriate fractions concentrated $\left(\mathrm{R}_{f}=0.3,20 \%\right.$ ethyl acetate/hexanes) to afford $76 \mathrm{mg}$ of the title compound in 70\% yield: IR (film) 3264, 3156, 2970, 2873, 1722, 1676, 1600, 1504, 1488, 1384, 1272 , 1180, 1151, 1104, $708 \mathrm{~cm}^{-1}$; ${ }^{1} \mathrm{H}-\mathrm{NMR}\left(600 \mathrm{MHz}, \mathrm{CDCl}_{3}\right) \delta 9.15(\mathrm{~s}, 1 \mathrm{H}), 7.90-7.91(\mathrm{~m}, 2 \mathrm{H}), 7.56-7.59$ $(\mathrm{m}, 1 \mathrm{H}), 7.46-7.49(\mathrm{~m}, 2 \mathrm{H}), 3.66(\mathrm{q}, J=7.2,1 \mathrm{H}), 1.24(\mathrm{~d}, J=7.2,6 \mathrm{H}) ;{ }^{13} \mathrm{C}-\mathrm{NMR}\left(100 \mathrm{MHz}, \mathrm{CDCl}_{3}\right) \delta$ 180.7, 165.5, 133.0, 132.9, 128.8, 127.8, 34.9, 18.7; HRMS (ES+): Exact mass calcd for $\mathrm{C}_{11} \mathrm{H}_{13} \mathrm{NO}_{2}$ $[\mathrm{M}+\mathrm{H}]^{+}:$192.1024. Found: 192.1016 .<smiles>CC(C)CC(=O)NC(=O)c1ccccc1</smiles>

N-(3-methylbutanoyl)benzamide (Table 1, Entry 3). 2-isobutyl-4,5-diphenyloxazole (122 mg, 0.44 mmol) was dissolved in $\mathrm{MeCN}(6.6 \mathrm{~mL})$ and $\mathrm{H}_{2} \mathrm{O}(0.73 \mathrm{~mL})$, and CAN (916 mgs, $\left.1.67 \mathrm{mmol}\right)$ was added subsequently. The reaction was allowed to stir at room temperature for 2 hours. After work up, the reaction was purified via flash chromatography (silica, $10 \% \rightarrow 25 \% \mathrm{v} / \mathrm{v}$ ethyl acetate/hexanes) and the appropriate fractions concentrated $\left(\mathrm{R}_{f}=0.3,20 \% \mathrm{v} / \mathrm{v}\right.$ ethyl acetate/hexanes) to afford $76 \mathrm{mg}$ of the title compound in 84\% yield: IR (film) 3279, 3073, 2957, 2870, 1708, 1686, 1506, 1469, 1390, 1372, 1302, 1241, 1197, 909, $707 \mathrm{~cm}^{-1}$; ${ }^{1} \mathrm{H}-\mathrm{NMR}\left(600 \mathrm{MHz}, \mathrm{CDCl}_{3}\right) \delta 9.21$ (s, 1H), 7.90-7.92 (m, 2H), 7.57$7.60(\mathrm{~m}, 1 \mathrm{H}), 7.47-7.50(\mathrm{~m}, 2 \mathrm{H}), 2.89(\mathrm{~d}, J=6.6 \mathrm{~Hz}, 2 \mathrm{H}), 2.24(\mathrm{~m}, 1 \mathrm{H}), 1.06(\mathrm{~d}, J=6.6 \mathrm{~Hz}) ;{ }^{13} \mathrm{C}-\mathrm{NMR}$ $\left(100 \mathrm{MHz}, \mathrm{CDCl}_{3}\right) \delta 176.1,165.7,133.1,132.8,128.9,127.8,46.2,24.7,22.5 ;$ HRMS (ES+): Exact mass calcd for $\mathrm{C}_{12} \mathrm{H}_{15} \mathrm{NO}_{2}[\mathrm{M}+\mathrm{H}]^{+}: 206.1181$. Found: 206.1190 .<smiles>O=C(NC(=O)C1CCCCC1)c1ccccc1</smiles>

$\mathrm{N}$-(cyclohexanecarbonyl)benzamide (Table 1, Entry 4). 2-cyclohexyl-4,5-diphenyloxazole (100 mg, $0.33 \mathrm{mmol})$ was dissolved in $\mathrm{MeCN}(5.0 \mathrm{~mL})$ and $\mathrm{H}_{2} \mathrm{O}(0.50 \mathrm{~mL})$, and CAN (687 $\left.\mathrm{mg}, 1.25 \mathrm{mmol}\right)$ was added subsequently. The reaction was allowed to stir at room temperature for 2 hours. After work up, the reaction was purified via flash chromatography (silica, $10 \% \rightarrow 25 \% \mathrm{v} / \mathrm{v}$ ethyl acetate/hexanes) and the appropriate fractions concentrated $\left(\mathrm{R}_{f}=0.4,20 \% \mathrm{v} / \mathrm{v}\right.$ ethyl acetate/hexanes) to afford $69 \mathrm{mgs}$ of the title compound in 91\% yield: IR (film) 3271, 3156, 3060, 2933, 2853, 1722, 1679, 1601, 1582, 1510, 
1486, 1450, 1273, 1242, 1156, 1133, 1074, 742, $706 \mathrm{~cm}^{-1} ;{ }^{1} \mathrm{H}-\mathrm{NMR}\left(600 \mathrm{MHz}, \mathrm{CDCl}_{3}\right) \delta 8.80(\mathrm{~s}, 1 \mathrm{H})$, 7.85-7.89 (m, 2H), 7.57-7.60 (m, 1H), 7.46-7.50 (m, 2H), 3.37-3.41 (m, 1H), 1.98-2.00 (m, 2H), 1.80$1.83(\mathrm{~m}, 2 \mathrm{H}), 1.71-1.75(\mathrm{~m}, 1 \mathrm{H}), 1.45-1.52(\mathrm{~m}, 2 \mathrm{H}), 1.35-1.42(\mathrm{~m}, 2 \mathrm{H}), 1.21-1.29(\mathrm{~m}, 1 \mathrm{H}) ;{ }^{13} \mathrm{C}-\mathrm{NMR}$ $\left(100 \mathrm{MHz}, \mathrm{CDCl}_{3}\right) \delta 179.2,165.4,133.1,133.0,128.9,127.7,44.5,28.9,25.9,25.6$; HRMS (ES+): Exact mass calcd for $\mathrm{C}_{14} \mathrm{H}_{17} \mathrm{NO}_{2}[\mathrm{M}+\mathrm{H}]^{+}: 232.1337$. Found: 232.1334 .<smiles>O=C(Cc1ccccc1)NC(=O)Cc1ccccc1</smiles>

$\mathrm{N}$-(2-phenylacetyl)benzamide (Table 1, Entry 5). 2-benzyl-4,5-diphenyloxazole (100 mg, 0.321 mmol) was dissolved in $\mathrm{MeCN}(4.8 \mathrm{~mL})$ and $\mathrm{H}_{2} \mathrm{O}(4.8 \mathrm{~mL})$, and CAN (669 mg, $1.22 \mathrm{mmol}$ ) was added subsequently. The reaction was allowed to stir at room temperature for 2 hours. After work up, the reaction was purified via HPLC ( $1 \% i$-PrOH/hexs) to afford $42 \mathrm{mg}$ of the title compound in $55 \%$ yield: IR (film) 3270, 3060, 2924, 1672, 1505, 1470, 1391, 1370, 1257, 1182, 1072, 1026, $698 \mathrm{~cm}^{-1}$; ${ }^{1} \mathrm{H}-$ NMR $\left(400 \mathrm{MHz}, \mathrm{CDCl}_{3}\right) \delta 8.95(\mathrm{~s}, 1 \mathrm{H}), 7.84(\mathrm{dd}, J=1.2$ and $6.8 \mathrm{~Hz}, 2 \mathrm{H}), 7.58(\mathrm{t}, J=8 \mathrm{~Hz}, 1 \mathrm{H}), 7.46$ (t, $J=8 \mathrm{~Hz}, 2 \mathrm{H}), 7.34-7.38(\mathrm{~m}, 5 \mathrm{H}), 4.34(\mathrm{~s}, 1 \mathrm{H}) ;{ }^{13} \mathrm{C}-\mathrm{NMR}\left(100 \mathrm{MHz}, \mathrm{CDCl}_{3}\right) \delta 173.9,165.5,133.7$, 133.3, 132.6, 129.8, 129.0, 128.6, 127.7, 127.2, 43.9; HRMS (ES+): Exact mass calcd for $\mathrm{C}_{15} \mathrm{H}_{13} \mathrm{NO}_{2}$ $[\mathrm{M}+\mathrm{H}]^{+}:$240.1205. Found: 240.1 .<smiles>O=C(NC(=O)c1ccccc1)C(=O)NC(=O)c1ccccc1</smiles>

$N$-pivaloylbenzamide (Table 1, Entry 6). 2-tert-butyl-4,5-diphenyloxazole (100 mg, $0.36 \mathrm{mmol}$ ) was dissolved in $\mathrm{MeCN}(5.4 \mathrm{~mL})$ and $\mathrm{H}_{2} \mathrm{O}(0.6 \mathrm{~mL})$, and $\mathrm{CAN}(750 \mathrm{mg}, 1.37 \mathrm{mmol})$ was added subsequently. The reaction was allowed to stir at room temperature for 2 hours. After work up, the reaction was purified via flash chromatography (silica, $10 \% \rightarrow 25 \% \mathrm{v} / \mathrm{v}$ ethyl acetate/hexanes) and the appropriate fractions concentrated $\left(\mathrm{R}_{f}=0.3,20 \% \mathrm{v} / \mathrm{v}\right.$ ethyl acetate/hexanes) to afford $66.5 \mathrm{mg}$ of the title compound in 90\% yield: IR (film) 3324, 3060, 2970, 2875, 1729, 1684, 1600, 1583, 1486, 1457, 1301, 1326, 1292, 1281, 1184, 1143, 1066, 1027, 847, $797 \mathrm{~cm}^{-1} ;{ }^{1} \mathrm{H}-\mathrm{NMR}\left(600 \mathrm{MHz}, \mathrm{CDCl}_{3}\right) \delta 8.81(\mathrm{~s}$, $1 \mathrm{H}), 7.76-7.77(\mathrm{~m}, 2 \mathrm{H}), 7.58-7.61(\mathrm{~m}, 1 \mathrm{H}), 7.48-7.50(\mathrm{~m}, 2 \mathrm{H}), 1.35(\mathrm{~s}, 9 \mathrm{H}) ;{ }^{13} \mathrm{C}-\mathrm{NMR}(100 \mathrm{MHz}$, $\left.\mathrm{CDCl}_{3}\right) \delta 176.2,166.7,133.7,132.7,130.0,128.6,128.3,127.6,40.5,26.9$; HRMS (ES+): Exact mass calcd for $\mathrm{C}_{12} \mathrm{H}_{15} \mathrm{NO}_{2}[\mathrm{M}+\mathrm{H}]^{+}$: 206.1181. Found: 206.1172. 
<smiles>O=CNC(=O)c1ccccc1</smiles>

$\boldsymbol{N}$-formylbenzamide (Table 1, Entry 7). 4,5-diphenyloxazole (103 $\mathrm{mg}, 0.466 \mathrm{mmol}$ ) was dissolved in MeCN (6.8 mL) and $\mathrm{H}_{2} \mathrm{O}(0.75 \mathrm{~mL})$, and CAN (867 mg, $\left.1.58 \mathrm{mmol}\right)$ was added subsequently. The reaction was allowed to stir at room temperature for 2 hours. After work up, the reaction was purified via flash chromatography ( silica, $10 \% \rightarrow 25 \% \mathrm{v} / \mathrm{v}$ ethyl acetate/hexanes) and the appropriate fractions concentrated $\left(\mathrm{R}_{f}=0.4,20 \% \mathrm{v} / \mathrm{v}\right.$ ethyl acetate/ hexanes) to afford $61.8 \mathrm{mg}$ of the title compound in $89 \%$ yield: IR (film) 3272, 1726, 1683, 1601, 1582, 1502, 1460, 1443, 1367, 1322, 1254, 1060, 884, 805, $752 \mathrm{~cm}^{-1} ;{ }^{1} \mathrm{H}-\mathrm{NMR}\left(600 \mathrm{MHz}, \mathrm{CDCl}_{3}\right) \delta 10.44(\mathrm{~d}, J=7.8 \mathrm{~Hz}, 1 \mathrm{H}), 9.44(\mathrm{~d}, J=9.6 \mathrm{Jz}, 1 \mathrm{H}), 8.05-8.07$ $(\mathrm{m}, 2 \mathrm{H}), 7.69-7.70(\mathrm{~m}, 1 \mathrm{H}), 7.57-7.64(\mathrm{~m}, 2 \mathrm{H}) ;{ }^{13} \mathrm{C}-\mathrm{NMR}\left(100 \mathrm{MHz}, \mathrm{CDCl}_{3}\right) \delta 166.8,165.2,133.9$, 131.0, 129.0, 128.1; HRMS (ES+): Exact mass calcd for $\mathrm{C}_{8} \mathrm{H}_{7} \mathrm{NO}_{2}[\mathrm{M}+\mathrm{H}]^{+}$: 150.0555. Found: 150.0555 .<smiles>O=C(/C=C/c1ccc(Cl)cc1)NC(=O)c1ccccc1</smiles>

(E)-N-(3-(4-chlorophenyl)acryloyl)benzamide (Table 1, Entry 8). (E)-2-(4-chlorostyryl)-4,5diphenyloxazole (50 mg, $0.14 \mathrm{mmol}$ ) was dissolved in $\mathrm{MeCN}(2.1 \mathrm{~mL})$ and $\mathrm{H}_{2} \mathrm{O}(0.23 \mathrm{~mL})$, and CAN (292 $\mathrm{mg}, 0.532 \mathrm{mmol}$ ) was added subsequently. The reaction was allowed to stir at room temperature for 2 hours. After work up, the reaction was purified via flash chromatography (silica, $10 \% \rightarrow 25 \% \mathrm{v} / \mathrm{v}$ ethyl acetate/hexanes) and the appropriate fractions concentrated $\left(\mathrm{R}_{f}=0.4,20 \% \mathrm{v} / \mathrm{v}\right.$ ethyl acetate/hexanes) to afford $39 \mathrm{mg}$ of the title compound in 98\% yield: IR (film) 3281, 3058, 1709, 1666, 1614, 1491, 1468, 1407, 1358, 1248, 1196, 1092, 979, 815, $695 \mathrm{~cm}^{-1} ;{ }^{1} \mathrm{H}-\mathrm{NMR}\left(600 \mathrm{MHz}, \mathrm{CDCl}_{3}\right) \delta$ $8.88(\mathrm{~s}, 1 \mathrm{H}), 7.91-7.92(\mathrm{~m}, 2 \mathrm{H}), 7.84(\mathrm{~d}, J=2.4 \mathrm{~Hz}, 2 \mathrm{H}), 7.63(\mathrm{t}, J=7.2 \mathrm{~Hz}, 1 \mathrm{H}), 7.56-7.58(\mathrm{~m}, 2 \mathrm{H})$, 7.51-7.54 (m, 2H), 7.37-7.38 (m, 2H); ${ }^{13} \mathrm{C}-\mathrm{NMR}\left(100 \mathrm{MHz}, \mathrm{CDCl}_{3}\right) \delta 167.5,165.9,145.2,136.6$, 133.4, 133.1, 132.9, 129.8, 129.2, 129.1, 127.8, 119.9; HRMS (ES+): Exact mass calcd for $\mathrm{C}_{16} \mathrm{H}_{12} \mathrm{ClNO}_{2}[\mathrm{M}+\mathrm{H}]^{+}:$286.0635. Found: 286.0643 .<smiles>C/C=C/C(=O)NC(=O)c1ccccc1</smiles> 
(E)-N-but-2-enoylbenzamide (Table 1, Entry 9). (E)-4,5-diphenyl-2-(prop-1-enyl)oxazole (100 mg, $0.383 \mathrm{mmol})$ was dissolved in $\mathrm{MeCN}(5.75 \mathrm{~mL})$ and $\mathrm{H}_{2} \mathrm{O}(0.64 \mathrm{~mL})$, and CAN (798 mg, $\left.1.46 \mathrm{mmol}\right)$ was added subsequently. The reaction was allowed to stir at room temperature for 2 hours. After work up, the reaction was purified via flash chromatography (silica, 10\% $\rightarrow 25 \% \mathrm{v} / \mathrm{v}$ ethyl acetate/hexanes) and the appropriate fractions concentrated $\left(\mathrm{R}_{f}=0.3,20 \% \mathrm{v} / \mathrm{v}\right.$ ethyl acetate/hexanes $)$ to afford $49 \mathrm{mg}$ of the title compound in 68\% yield: IR (film) 3264, 3068, 2923, 2857, 1725, 1679, 1644, 1601, 1582, $1504,1484,1442,1337,1262,1208,1168,1106,1073,1028,968,915,866,832,799,707 \mathrm{~cm}^{-1}$; ${ }^{1} \mathrm{H}-$ NMR $\left(600 \mathrm{MHz}, \mathrm{CDCl}_{3}\right) \delta 8.66(\mathrm{~s}, 1 \mathrm{H}), 7.90-7.92(\mathrm{~m}, 2 \mathrm{H}), 7.64-7.66(\mathrm{~m}, 1 \mathrm{H}), 7.53-7.56(\mathrm{~m}, 2 \mathrm{H}), 7.19-$ $7.30(\mathrm{~m}, 2 \mathrm{H}), 2.03(\mathrm{~d}, J=6.6 \mathrm{~Hz}, 3 \mathrm{H}) ;{ }^{13} \mathrm{C}-\mathrm{NMR}\left(100 \mathrm{MHz}, \mathrm{CDCl}_{3}\right) \delta 167.3,165.8,147.3,133.2$, 133.0, 129.0, 127.7, 124.1, 18.6; HRMS (ES+): Exact mass calcd for $\mathrm{C}_{11} \mathrm{H}_{11} \mathrm{NO}_{2}[\mathrm{M}+\mathrm{H}]^{+}: 190.0868$. Found: 190.0872 .<smiles>O=C1C=CC(O)N1C(=O)c1ccccc1</smiles>

1-benzoyl-5-hydroxy-1H-pyrrol-2(5H)-one (Table 1, Entry 10). (E)-3-(4,5-diphenyloxazol-2yl)acrylaldehyde ${ }^{\text {Error! Bookmark not defined. }}(97.1 \mathrm{mg}, 0.353 \mathrm{mmol})$ was dissolved in MeCN (5.3 mL) and $\mathrm{H}_{2} \mathrm{O}(0.6 \mathrm{~mL})$, and CAN (735 mg, $1.34 \mathrm{mmol}$ ) was added subsequently. The reaction was allowed to stir at room temperature for 2 hours. After work up, the reaction was purified via flash chromatography (silica, $10 \% \rightarrow 30 \% \mathrm{v} / \mathrm{v}$ ethyl acetate/hexanes) and the appropriate fractions concentrated $\left(\mathrm{R}_{f}=0.1\right.$, $15 \% \mathrm{v} / \mathrm{v}$ ethyl acetate/hexanes) to afford $47.2 \mathrm{mg}$ of the title compound in $66 \%$ yield: IR (film) 3412 , $3105,2919,1732,1670,1601,1492,1448,1322,1309,1195,1128,109,1044,995,905 \mathrm{~cm}^{-1} ;{ }^{1} \mathrm{H}-$ NMR (500MHz, $\left.\mathrm{CDCl}_{3}\right) \delta$ 7.64-7.65 (m, 2H), 7.54-7.57 (m, 1H), 7.42-7.45 (m, 2H), $7.19(\mathrm{dd}, J=6 \mathrm{~Hz}$, $1 \mathrm{~Hz}, 1 \mathrm{H}), 6.39(\mathrm{~s}, 1 \mathrm{H}), 6.18(\mathrm{dd}, J=6 \mathrm{~Hz}, 1 \mathrm{~Hz}), 4.74(\mathrm{~s}, 1 \mathrm{H}) ;{ }^{13} \mathrm{C}-\mathrm{NMR}\left(100 \mathrm{MHz}, \mathrm{CDCl}_{3}\right) \delta 170.0$, 166.8, 147.7, 133.4, 132.4, 129.0, 128.1, 127.8, 82.2; HRMS (ES+): Exact mass calcd for $\mathrm{C}_{11} \mathrm{H}_{9} \mathrm{NO}_{3}$ $[\mathrm{M}+\mathrm{H}]^{+}:$204.0660. Found: 204.0667.

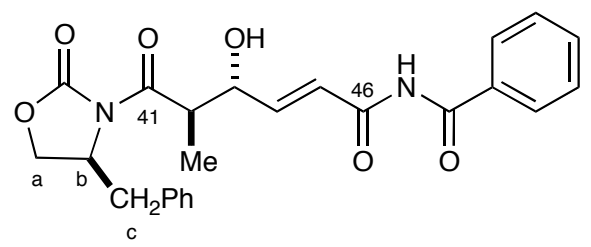

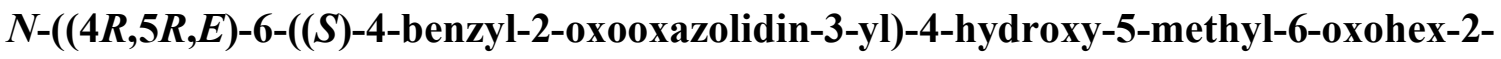


enoyl)benzamide (Table 1, Entry 11). To the pre-cooled to $0{ }^{\circ} \mathrm{C}$ solution of $(S)$-4-benzyl-3$\left((2 R, 3 R, E)\right.$-5-(4,5-diphenyloxazol-2-yl)-3-hydroxy-2-methylpent-4-enoyl)oxazolidin-2-one ${ }^{2}(50.5 \mathrm{mg}$, $98.8 \mathrm{mmol})$ in acetonitrile/water mixture $(7.5: 1,1.7 \mathrm{~mL}) \mathrm{CAN}(115 \mathrm{mg}, 0.211 \mathrm{mmol})$ was added. The obtained orange solution was stirred for $1 \mathrm{~h}$, and then another portion of CAN (16 mg, $0.030 \mathrm{mmol})$ was introduced. The reaction mixture was stirred for additional 30 minutes before being treated with water $(4 \mathrm{~mL})$ and ethyl acetate $(5 \mathrm{~mL})$. The aqueous phase was extracted with ethyl acetate $(2 \times 2 \mathrm{~mL})$, and the combined organic phase was washed with brine $(3 \mathrm{~mL})$, dried $\left(\mathrm{MgSO}_{4}\right)$, filtered and concentrated under reduced pressure to afford a white solid. The purification of the crude product by flash chromatography (silica, 33\% $\rightarrow 50 \% \mathrm{v} / \mathrm{v}$ ethyl acetate/hexanes elution) afforded, after concentration of the appropriate fractions $\left(\mathrm{R}_{f} 0.4,50 \% \mathrm{v} / \mathrm{v}\right.$ ethyl acetate/hexanes), the title compound $(26.0 \mathrm{mg}, 60 \%)$ as a white foam: $[\alpha]_{\mathrm{D}}{ }^{25}=+35.1\left(c=0.75, \mathrm{CHCl}_{3}\right)$; IR (film) $3476,3322,3025,2970$, 2926, 2849, 1746, 1737, 1704, 1682, 1654, 1638, 1561, 1501, 1473, 1385, 1352, 1325, 1242, 1209, 1181, 1022, $978 \mathrm{~cm}^{-1} ;{ }^{1} \mathrm{H}$ NMR (500 MHz, $\mathrm{CDCl}_{3}$ ) $\delta 8.70$ (s, NH), 7.83 (dd, $J=8.4,1.2 \mathrm{~Hz}, 2 \mathrm{H}$ ), 7.59 (tt, $J=7.8,1.2 \mathrm{~Hz}, 1 \mathrm{H}), 7.48$ (t, $J=7.8 \mathrm{~Hz}, 2 \mathrm{H}), 7.39$ (dd, $J=15.8,1.5 \mathrm{~Hz}, 1 \mathrm{H}), 7.32$ (t, $J=7.5 \mathrm{~Hz}$, 2H), 7.26 (t, $J=7.8 \mathrm{~Hz}, 1 \mathrm{H}), 7.23(\mathrm{dd}, J=15.5,5.4 \mathrm{~Hz}, 1 \mathrm{H}), 7.20$ (d, $J=7.2 \mathrm{~Hz}, 2 \mathrm{H}), 4.69$ (ddt, $J=$ 10.8, 7.6, 3.2 Hz, 1H), $4.56(\mathrm{~m}, 1 \mathrm{H}), 4.21(\mathrm{t}, J=8.2 \mathrm{~Hz}, 1 \mathrm{H}), 4.18$ (dd, $J=9.1,2.9 \mathrm{~Hz}, 1 \mathrm{H}), 4.12$ (p, $J$ $=6.9 \mathrm{~Hz}, 1 \mathrm{H}), 3.29(\mathrm{dd}, J=13.6,3.4 \mathrm{~Hz}, 1 \mathrm{H}), 2.77(\mathrm{dd}, J=13.5,9.7 \mathrm{~Hz}, 1 \mathrm{H}), 1.33(\mathrm{~d}, J=7.03 \mathrm{H}) ;{ }^{13} \mathrm{C}$ NMR (125 MHz, $\left.\mathrm{CDCl}_{3}\right) \delta 175.8,166.8,165.6,153.3,149.0,135.1,133.3,132.8,129.5,129.0,129.0$, 127.7, 123.7, 77.2, 74.0, 66.2, 55.5, 42.6, 37.8, 14.6; HRMS (ES+): Exact mass calcd for $\mathrm{C}_{24} \mathrm{H}_{24} \mathrm{~N}_{2} \mathrm{O}_{6}$ $[\mathrm{M}+\mathrm{H}]^{+}:$437.1712. Found: 437.1700 .

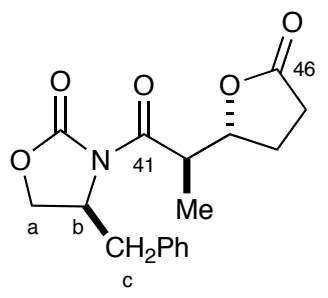

(S)-4-benzyl-3-((R)-2-((R)-5-oxo-tetrahydrofuran-2-yl)propanoyl)oxazolidin-2-one (Table 1, Entry 12). To the pre-cooled to $0{ }^{\circ} \mathrm{C}$ solution of (S)-4-benzyl-3-((2R,3R)-5-(4,5-diphenyloxazol-2-yl)3-hydroxy-2-methylpentanoyl)oxazolidin-2-one ${ }^{2}(77.0 \mathrm{mg}, 151 \mathrm{mmol})$ in acetonitrile/water mixture (10:1, $2.6 \mathrm{~mL})$ CAN (214 mg, $392 \mathrm{mmol}$ ) was added. The obtained orange solution was stirred for 2.5 $\mathrm{h}$ before being treated with water $(5 \mathrm{~mL})$ and ethyl acetate $(5 \mathrm{~mL})$. The aqueous phase was extracted with ethyl acetate $(2 \times 2 \mathrm{~mL})$, and the combined organic phase was washed with brine $(3 \mathrm{~mL})$, dried 
$\left(\mathrm{MgSO}_{4}\right)$, filtered and concentrated under reduced pressure to afford a white solid. The purification of the crude product by flash chromatography (silica, $50 \% \rightarrow 75 \% \mathrm{v} / \mathrm{v}$ ethyl acetate/hexanes elution) afforded, after concentration of the appropriate fractions $\left(\mathrm{R}_{f} 0.2,50 \% \mathrm{v} / \mathrm{v}\right.$ ethyl acetate/hexanes), the title compound (39.0 mg, $81 \%)$ as a white foam: $[\mathrm{a}]_{\mathrm{D}}{ }^{25}=+13.3\left(c=1.80, \mathrm{CHCl}_{3}\right)$; IR (film) 3081 , 2982, 2927, 1773, 1702, 1698, 1680, 1560, 1390, 1362, 1275, 1220, 1177, 1133, 1111, 1067, 1023, $979 \mathrm{~cm}^{-1} ;{ }^{1} \mathrm{H}$ NMR $\left(500 \mathrm{MHz}, \mathrm{CDCl}_{3}\right) \delta 7.34(\mathrm{t}, J=7.3 \mathrm{~Hz}, 2 \mathrm{H}), 7.27(\mathrm{t}, J=7.1 \mathrm{~Hz}, 1 \mathrm{H}), 7.24(\mathrm{~d}, J=$ $7.3 \mathrm{~Hz}, 2 \mathrm{H}), 4.80$ (dt, $J=9.3,7.3 \mathrm{~Hz}, 1 \mathrm{H}), 4.70(\mathrm{ddt}, J=9.3,6.8,3.4 \mathrm{~Hz}, 1 \mathrm{H}), 4.23-4.18(\mathrm{~m}, 2 \mathrm{H})$, $4.14(\mathrm{dq}, J=9.3,6.8 \mathrm{~Hz}, 1 \mathrm{H}), 3.31(\mathrm{dd}, J=13.7,3.4 \mathrm{~Hz}, 1 \mathrm{H}), 2.85(\mathrm{dd}, J=13.5,9.3 \mathrm{~Hz}, 1 \mathrm{H}), 2.63$ (ddd, $J=17.6,9.8,4.9 \mathrm{~Hz}, 1 \mathrm{H}$ ), 2.56 (dt, $J=18.0,9.3 \mathrm{~Hz}, 1 \mathrm{H}$ ), 2.41 (dddd, $J=12.7,9.3,6.8,4.9 \mathrm{~Hz}$, $1 \mathrm{H}), 2.03(\mathrm{ddt}, J=12.5,9.3,7.8 \mathrm{~Hz}, 1 \mathrm{H}), 1.21(\mathrm{~d}, J=6.83 \mathrm{H}) ;{ }^{13} \mathrm{C} \mathrm{NMR}\left(125 \mathrm{MHz}, \mathrm{CDCl}_{3}\right) \delta 176.2$, 174.0, 153.2, 135.1, 129.5, 129.0, 127.3, 81.3, 66.1, 55.4, 42.5, 37.6, 28.2, 25.8, 12.9; HRMS (ES+): Exact mass calcd for $\mathrm{C}_{17} \mathrm{H}_{19} \mathrm{NO}_{5}\left[\mathrm{M}+\mathrm{NH}_{4}\right]^{+}: 335.1607$. Found: 335.1602 .<smiles>O=C(NC(=O)c1ccccc1)c1ccccc1</smiles>

$\boldsymbol{N}$-benzoylbenzamide (Table 3, Entries 1, 2). 2,4-diphenyloxazole (50 $\mathrm{mg}, 0.226 \mathrm{mmol}$ ) was dissolved in $\mathrm{MeCN}(3.4 \mathrm{~mL})$ and $\mathrm{H}_{2} \mathrm{O}(0.37 \mathrm{~mL})$, and $\mathrm{CAN}(471 \mathrm{mg}, 0.859 \mathrm{mmol})$ was added subsequently. The reaction was allowed to stir at room temperature for 2 hours. After work up, the reaction was purified via flash chromatography (silica, $10 \% \rightarrow 25 \% \mathrm{v} / \mathrm{v}$ ethyl acetate/hexanes) and the appropriate fractions concentrated ( $\mathrm{R}_{f}=0.6,20 \% \mathrm{v} / \mathrm{v}$ ethyl acetate/hexanes) to afford $31.7 \mathrm{mg}$ of the title compound in 93\% yield: IR (film) 3249, 3059, 2923, 1706, 1676, 1600, 1502, 1476, 1225, 1177, 1116, 1072, 1025, 955, $806 \mathrm{~cm}^{-1}$; ${ }^{1} \mathrm{H}-\mathrm{NMR}\left(600 \mathrm{MHz}, \mathrm{CDCl}_{3}\right) \delta 9.14(\mathrm{~s}, 1 \mathrm{H}), 7.85-7.86(\mathrm{~m}, 4 \mathrm{H}), 7.58-$ $7.60(\mathrm{~m}, 2 \mathrm{H}), 7.47-7.50(\mathrm{~m}, 4 \mathrm{H}) ;{ }^{13} \mathrm{C}-\mathrm{NMR}\left(100 \mathrm{MHz}, \mathrm{CDCl}_{3}\right) \delta 166.8,133.6,133.3,129.1,128.2$; HRMS (ES+): Exact mass calcd for $\mathrm{C}_{14} \mathrm{H}_{11} \mathrm{NO}_{2}[\mathrm{M}+\mathrm{H}]^{+}: 226.0868$. Found: 226.0867.<smiles>CC(=O)NC(=O)c1ccccc1</smiles>

$\mathrm{N}$-acetylbenzamide (Table 3, Entry 3). To the solution of 2,5-dimethyl-4-phenyloxazole (31.0 $\mathrm{mg}$, $191 \mathrm{mmol})$ in acetonitrile/water mixture $(9: 1,2.0 \mathrm{~mL})$ CAN $(344 \mathrm{mg}, 639 \mathrm{mmol})$ was added. The obtained solution was stirred for $30 \mathrm{~min}$ before being treated with water $(5 \mathrm{~mL})$ and ethyl acetate $(5$ $\mathrm{mL})$. The aqueous phase was extracted with ethyl acetate $(2 \times 3 \mathrm{~mL})$, and the combined organic phase 
was washed with brine $(3 \mathrm{~mL})$, dried $\left(\mathrm{MgSO}_{4}\right)$, filtered and concentrated under reduced pressure to afford an orange solid. The purification of the crude product by flash chromatography (silica, $33 \% \mathrm{v} / \mathrm{v}$ ethyl acetate/hexanes elution) afforded, after the concentration of the appropriate fractions $\left(\mathrm{R}_{f} 0.4,33 \%\right.$ $\mathrm{v} / \mathrm{v}$ ethyl acetate/hexanes), the title compound (28.0 mg, 90\%) as a white solid: IR (film) 3272, 3166, 3060, 2954, 2919, 2849, 1713, 1689, 1602, 1502, 1478, $1243 \mathrm{~cm}^{-1} ;{ }^{1} \mathrm{H}$ NMR $\left(500 \mathrm{MHz}, \mathrm{CDCl}_{3}\right) \delta 8.97$ (s, NH), $7.87(\mathrm{~d}, J=6.9 \mathrm{~Hz}, 2 \mathrm{H}), 7.60(\mathrm{t}, J=7.3 \mathrm{~Hz}, 1 \mathrm{H}), 7.50(\mathrm{t}, J=6.9 \mathrm{~Hz}, 2 \mathrm{H}), 2.61(\mathrm{~s}, 3 \mathrm{H}) ;{ }^{13} \mathrm{C}$ NMR (125 MHz, $\left.\mathrm{CDCl}_{3}\right) \delta$ 173.7, 165.7, 133.3, 132.7, 129.0, 127.7, 25.6; HRMS (ES+): Exact mass calcd for $\mathrm{C}_{9} \mathrm{H}_{9} \mathrm{NO}_{2}[\mathrm{M}+\mathrm{H}]^{+}$: 164.0711. Found: 164.0714.<smiles>CCOC(=O)C(=O)NC(=O)/C=C/c1ccccc1</smiles>

Ethyl 2-cinnamamido-2-oxoacetate (Table 3, Entry 4). (E)-ethyl 2-styryloxazole-4-carboxylate (108 $\mathrm{mg}, 0.444 \mathrm{mmol})$ was dissolved in $\mathrm{MeCN}(6.66 \mathrm{~mL})$ and $\mathrm{H}_{2} \mathrm{O}(0.74 \mathrm{~mL})$, and CAN (949 mg, 1.73 mmol) was added subsequently. The reaction was allowed to stir at room temperature for 2 hours. After work up, the reaction was purified via flash chromatography (silica, 10\% $\rightarrow 25 \% \mathrm{v} / \mathrm{v}$ ethyl acetate/hexanes) and the appropriate fractions concentrated $\left(\mathrm{R}_{f}=0.5,20 \% \mathrm{v} / \mathrm{v}\right.$ ethyl acetate/hexanes) to afford $102 \mathrm{mg}$ of the title compound in 93\% yield: IR (film) 3360, 3201, 2918, 2849, 1738, 1724, 1665, 1631, 1600, 1494, 1450, 1410, 1321, 1200, $967 \mathrm{~cm}^{-1}$; ${ }^{1} \mathrm{H}-\mathrm{NMR}\left(600 \mathrm{MHz}, \mathrm{CDCl}_{3}\right) \delta 9.29(\mathrm{~s}, 1 \mathrm{H}), 7.92$ $(\mathrm{d}, J=16.2 \mathrm{~Hz}, 1 \mathrm{H}), 7.60-7.61(\mathrm{~m}, 2 \mathrm{H}), 7.53-7.57(\mathrm{~m}, 1 \mathrm{H}), 7.39-7.42(\mathrm{~m}, 3 \mathrm{H}) 4.42(\mathrm{q}, J=7.2,2 \mathrm{H})$, $1.42(\mathrm{t}, J=7.2,3 \mathrm{H}) ;{ }^{13} \mathrm{C}-\mathrm{NMR}\left(100 \mathrm{MHz}, \mathrm{CDCl}_{3}\right) \delta 165.5,159.6,148.1,134.2,131.1,129.0,128.8$, 118.0, 110.7, 64.3, 13.9; HRMS (ES+): Exact mass calcd for $\mathrm{C}_{13} \mathrm{H}_{13} \mathrm{NO}_{4}[\mathrm{M}+\mathrm{H}]^{+}: 248.0923$. Found: 248.0913.

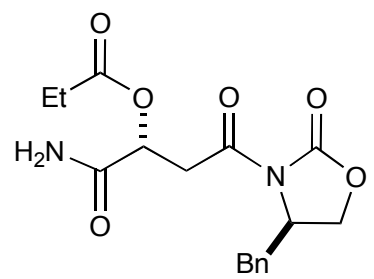

(R)-1-amino-4-((R)-4-benzyl-2-oxooxazolidin-3-yl)-1,4-dioxobutan-2-yl propionate (Table 3, Entry 5). ( $R$ )-3-((R)-4-benzyl-2-oxooxazolidin-3-yl)-1-(2-methyloxazol-4-yl)-3-oxopropyl propionate (50 mg, $0.13 \mathrm{mmol})$ was dissolved in $\mathrm{MeCN}(2.00 \mathrm{~mL})$ and $\mathrm{H}_{2} \mathrm{O}(0.20 \mathrm{~mL})$, and CAN (270 mg, 0.49 mmol) was added subsequently. The reaction was allowed to stir at room temperature overnight. The following day, more CAN (270 $\mathrm{mg}, 0.49 \mathrm{mmol})$ was added to drive the reaction to completion. After 
work up, the reaction was purified via flash chromatography (silica, $40 \% \rightarrow 70 \% \mathrm{v} / \mathrm{v}$ ethyl acetate/hexanes) and the appropriate fractions concentrated $\left(\mathrm{R}_{f}=0.2,50 \%\right.$ EtOAc/hexs $)$ to afford 30.5 $\mathrm{mg}$ of the title compound in $65 \%$ yield: $[\alpha]_{\mathrm{D}}{ }^{25}=-58\left(c=0.33, \mathrm{CHCl}_{3}\right)$; IR (film) $3450,3351,3192$, 2924, 1778, 1746, 1694, 1603, 1454, 1392, 1359, 1284, 1209, 1180, 1085, $989 \mathrm{~cm}^{-1}$; ${ }^{1} \mathrm{H}-\mathrm{NMR}$ $\left(500 \mathrm{MHz}, \mathrm{CDCl}_{3}\right) \delta$ 7.32-7.35 (m, 2H), 7.28-7.30 (m, 1H), 7.19-7.22 (m, 2H), $6.23(\mathrm{~s}, 1 \mathrm{H}), 5.66(\mathrm{t}, J=$ $6 \mathrm{~Hz}, 1 \mathrm{H}), 5.58(\mathrm{~s}, 1 \mathrm{H}), 4.64-4.68(\mathrm{~m}, 1 \mathrm{H}), 4.22-4.26(\mathrm{~m}, 1 \mathrm{H}), 4.18-4.20(\mathrm{~m}, 1 \mathrm{H}), 3.64(\mathrm{dd}, J=5.5 \mathrm{~Hz}$, $13 \mathrm{~Hz}, 1 \mathrm{H}$ ), 3.49 (dd, $J=6 \mathrm{~Hz}, 18 \mathrm{~Hz}, 1 \mathrm{H}$ ), 3.29 (dd, $J=3.5 \mathrm{~Hz}, 13.5 \mathrm{~Hz}, 1 \mathrm{H}$ ), 2.78 (dd, $J=4 \mathrm{~Hz}, 9.5$ $\mathrm{Hz}, 1 \mathrm{H}), 2.45(\mathrm{q}, J=8 \mathrm{~Hz}, 2 \mathrm{H}), 1.19(\mathrm{t}, J=8 \mathrm{~Hz}, 3 \mathrm{H}) ;{ }^{13} \mathrm{C}-\mathrm{NMR}\left(100 \mathrm{MHz}, \mathrm{CDCl}_{3}\right) \delta 173.3,171.1$, 169.3, 135.0, 129.4, 129.0, 128.4, 127.5, 69.0, 66.5, 55.2, 37.8, 37.7, 27.5, 8.9; HRMS (ES+): Exact mass calcd for $\mathrm{C}_{17} \mathrm{H}_{20} \mathrm{~N}_{2} \mathrm{O}_{6}[\mathrm{M}+\mathrm{H}]^{+}$: 348.1321. Found: 348.1 .

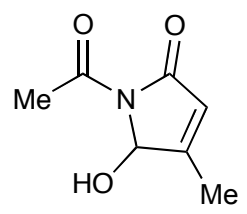

1-acetyl-5-hydroxy-4-methyl-1H-pyrrol-2(5H)-one (Table 3, Entry 6). (E)-2-methyl-3-(2methyloxazol-4-yl)acrylaldehyde $(50 \mathrm{mg}, 0.33 \mathrm{mmol})$ was dissolved in $\mathrm{MeCN}(5.00 \mathrm{~mL})$ and $\mathrm{H}_{2} \mathrm{O}$ $(0.50 \mathrm{~mL})$, and CAN (689 $\mathrm{mg}, 1.25 \mathrm{mmol})$ was added subsequently. The reaction was allowed to stir at room temperature for 2 hours. After work up, the reaction was purified via flash chromatography (silica, $10 \% \rightarrow 35 \% \mathrm{v} / \mathrm{v}$ ethyl acetate/hexanes) and the appropriate fractions concentrated $\left(\mathrm{R}_{f}=0.2\right.$, $25 \% \mathrm{v} / \mathrm{v}$ ethyl acetate/hexanes) to afford $28.2 \mathrm{mg}$ of the title compound in 55\% yield: IR (film) 3359 , 2920, 2852, 1732, 1677, 1385, 1348, 1326, 1115, 1077, $849 \mathrm{~cm}^{-1} ;{ }^{1} \mathrm{H}-\mathrm{NMR}\left(500 \mathrm{MHz}, \mathrm{CDCl}_{3}\right) \delta 5.87-$ $5.89(\mathrm{~m}, 2 \mathrm{H}), 4.34(\mathrm{~d}, J=6 \mathrm{~Hz}, 1 \mathrm{H}), 2.53(\mathrm{~s}, 1 \mathrm{H}), 2.12(\mathrm{~d}, J=1 \mathrm{~Hz}, 3 \mathrm{H}) ;{ }^{13} \mathrm{C}-\mathrm{NMR}\left(100 \mathrm{MHz}, \mathrm{CDCl}_{3}\right)$ $\delta$ 171.4, 168.0, 160.6, 122.8, 83.1, 24.3, 13.9; HRMS (ES+): Exact mass calcd for $\mathrm{C}_{7} \mathrm{H}_{9} \mathrm{NO}_{3}[\mathrm{M}+\mathrm{H}]^{+}$: 156.0660. Found: 156.0659 . 